\title{
Chasing the right path: tips, tricks and challenges of robotic approach to posterior segments
}

\author{
Fabrizio Di Benedetto, Giuseppe Tarantino, Paolo Magistri \\ Hepato-Pancreato-Biliary Surgery and Liver Transplantation Unit, University of Modena and Reggio Emilia, Modena, MO, Italy \\ Correspondence to: Dr. Paolo Magistri, MD. Hepato-Pancreato-Biliary Surgery and Liver Transplantation Unit University of Modena and Reggio \\ Emilia, Via del Pozzo 71, 41124 Modena, MO, Italy. Email: paolo.magistri@unimore.it.
}

Submitted Mar 29, 2019. Accepted for publication Apr 10, 2019.

doi: 10.21037/hbsn.2019.04.07

View this article at: http://dx.doi.org/10.21037/hbsn.2019.04.07

The advent of minimally invasive technologies to approach liver diseases changed some paradigms of liver surgery during the last two decades. They allow to safely treat patients with poor liver function, portal hypertension and cirrhosis, reducing the risk of decompensation (1). However, those potential benefits resulted to be in contrast with the actual application of such technologies in the real-life $(2,3)$. Moving from traditional open to laparoscopic surgery initially implied to rethink operative strategies and forced the first generation of minimally invasive surgeons to learn again several technical skills. For example, range of motion suddenly reduced from the seven degrees of freedom of the human hand to four, and physiologic tremor turned out to be amplified by the laparoscopic instrumentation, which was also poor in ergonomics. Moreover, suturing, knot tying and bimanual tissue manipulation, while considered basic surgical skills in open surgery, suddenly became advanced surgical skills in laparoscopy, requiring appropriate training and a relatively long learning curve (4). It has been largely described how robotic surgery represents a refinement of laparoscopy thanks to tremor filtration, three-dimensional stable view and endo-wristed instruments, but it still does not overcome some limitations of minimally invasive techniques proficiency gaining. Even when operating with the robot, surgeons still lack the integration between visual and manual control on the tissue. In other words, is not just the lack of haptic feedback that matters, but the condition of operating without the simultaneous info coming from visual and tactile senses, conveying data to the brain that are translated into a more complex interpretation of the tissues' characteristics. Organ manipulation and in particular liver mobilization is still an issue and the use of gravity to increase exposure is limited by the docking of the operative chart. The surgery of the liver poses additional technical difficulties due to the peculiar anatomy, the large surface and the high rate of vascular variations. Several scoring systems have been reported to stratify the difficulty and the risk of complications after liver resections with minimally invasive approach, based on lesions position and size $(5,6)$. Posterior segments represent a challenge due to convex surface of the liver, the difficulty to achieve an appropriate exposure and, therefore, the increased risk of bleeding and bile leakage. In this setting, the performances of the robot are really maximized thanks to both the easier suturing and the ability to reach deep spaces. However, it is crucial to approach this kind of resection having a clear knowledge of the anatomy and an appropriate pre-operative imaging study. In general, when approaching posterior segments robotically, patients should be positioned supine, about $20^{\circ}$ anti-Trendelenburg, rotated to the left and with a cushion under the right flank to enhance the access (Figure 1). As a general rule, trocars should be positioned very high subcostal and lateral, however their disposition may vary according to each patient peculiar conformation and lesion localization, and to the robotic platform in use. The goal is to create an adequate triangulation with enough space $(8 \mathrm{~cm}$ on average) between the ports (7). The fourth robotic arm is generally positioned on the left side. Trocar placement and docking have been considered a crucial and time consuming step at the beginning of the robotic experience, mainly due to the rigidity of the system that did not allow changing the position of the patient after having docked the cart (8). Nowadays, using the fourth generation of the robotic platform, we can find novel features such as a laser targeting system that makes the robotic arm set-up easier, and integrated Table Motion that allows dynamic 

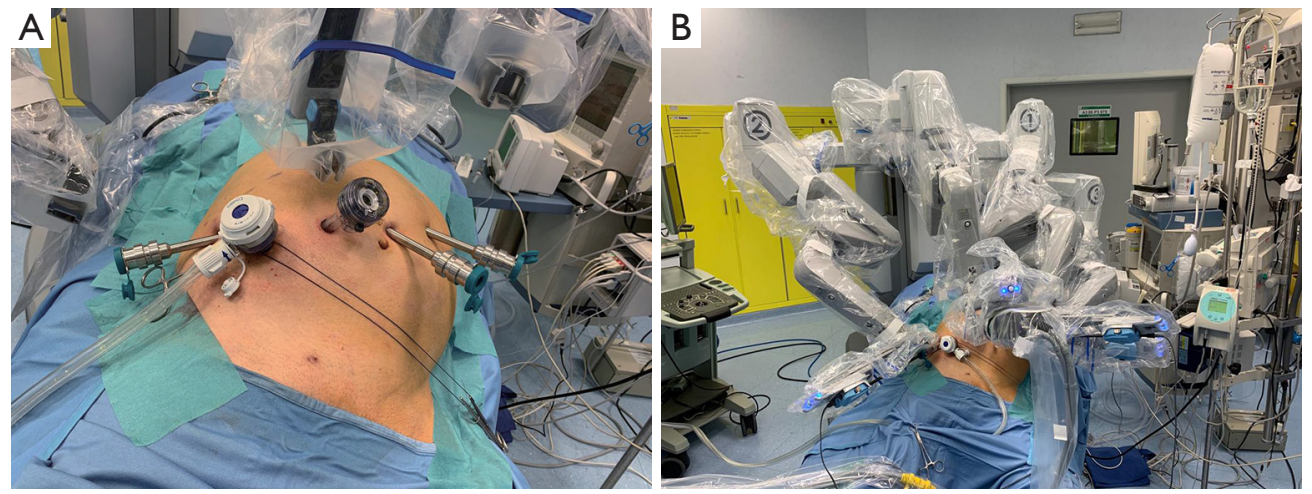

Figure 1 Disposition of the trocars (A) and docking of the robotic daVinci Si platform chart (B) for a segmentectomy of SVII.

repositioning of the patient while the surgeon operates without undocking. The Tile-Pro function on the robotic console is one of the most important resources of the platform and displays at the same time the classical endoscopic view and imaging sources such as the US, CT scan, MR images or $3 \mathrm{D}$ reconstructed models (9). Up to three different images can be viewed at the same time by the console surgeon and the operating room personnel. This represents a valuable improvement compared to standard laparoscopy since switching from a screen to another to visualize pre- and intra-operative imaging is no longer required. Another option to assess the correct transection line is to temporally close the portal branch with a minimally invasive vascular clamp (Bulldog) to identify the vascular demarcation. Parenchymal transection can be performed with a combination of monopolar and bipolar energy, and with the use of $\mathrm{daVinci}^{\circledR}$ Harmonic ACE $^{\mathrm{TM}}$ (Ethicon, Somerville, NJ, USA) for deeper layers. Although the robotic platform still does not support liverspecific articulated devices for parenchyma dissection such as the Cavitron Ultrasonic Surgical Aspirator (CUSA), the correct use of the available tools allows a safe dissection. For example, the Maryland forceps can be used as a right-angle to precisely dissect small vessels in the parenchyma thanks to the 7 degrees of freedom of the robotic instruments. The use of the bipolar electrosurgical instrument, namely daVinci ${ }^{\circledR}$ Vessel Sealer, is another option for vascular control. The technique to safely approach a right hepatectomy includes few precautions that are not amendable: the optical trocar must be on the mid-clavicular line to obtain an optimal and safe view of the vena cava during the entire procedure; first step should be the hilar dissection and control of the right branch of the portal vein and right hepatic artery, together with dissection of the hepatic veins at the caval confluence, to gain an easier control in case of emergency conditions; after the section of coronary and triangular right ligaments, the mobilization of the right lobe should continue on a bottom to up direction along the vena cava, holding the lobe with the fourth arm, to reduce the risk of lesions during parenchymal transection. Finally, the management of the right hepatic vein during right hepatectomies often cannot benefit from a selective dissection like in the left lobe, requiring trans-parenchymal control with a laparoscopic or robotic stapler. Similarly, right lateral sectionectomies require an optimal view to keep a straight transection plane. Segmentectomies of segments seven and eight are the most challenging procedures. In standard open surgery the left hand of the operating surgeon (or the assistant surgeon) holds and turns the right lobe to achieve a flat exposure of the upper right surface of the liver. This kind of movement is not reproducible in minimally invasive surgery, but the endowristed robotic instruments helps to retract the edge of the resection plane and to progressively transect the parenchyma without the need of a L-shaped trocar disposition like in the laparoscopic diamond technique (Figure 2) (10). It is advisable to prepare the operative field at the beginning of the procedure for an eventual Pringle maneuver, in order to have an easy control of the inflow in case of emergency. We usually put an additional trocar for the Pringle and we pass the hilum with an umbilical tape that is controlled with a chest tube to compress the hilum from outside.

Control of intraoperative bleeding is one of the most important advantages of the robotic platform. When experiencing a major bleeding or difficulty in controlling a vessel you can stop the hemorrhage with one of the robotic arms and plan the strategy with the rest of the team, or 

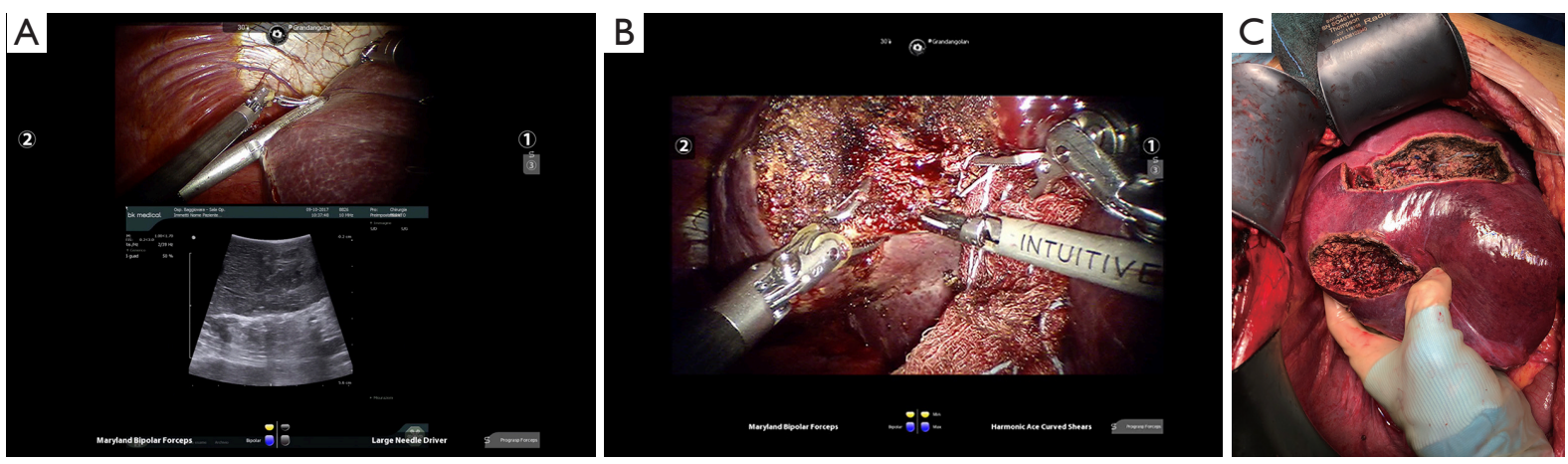

Figure 2 Robotic resection of segment VII: intraoperative ultrasound after mobilization of the right lobe (A), and role of the flexible instruments to reach deep spaces (B), in particular the fourth arm; (C) is intended to compare this kind of exposure with the open approach: the left hand of the operating surgeon is crucial to obtain a flat exposure of the resection plane.

even prepare the OR for a conversion to laparotomy, in a safe and calm way. In fact, the arm will keep holding the vessel and stopping the bleeding until you reactivate it. In our experience robotic approach increased the ability to resect posterior liver segments with minimally invasive approach, and in particular in cirrhotic patients, with excellent results in terms of intra-operative results and oncologic accuracy, post-operative morbidity and return to daily-life activity. However, the route to gaining proficiency in robotic skills has yet to be defined, and consensus on robotic surgery is still lacking. Therefore, implementation and development of this technology should be reserved to high volume HPB centers to guarantee patient safety and reliability of the innovation.

\section{Acknowledgments}

None.

\section{Footnote}

Conflicts of Interest: The authors have no conflicts of interest to declare.

\section{References}

1. Magistri P, Tarantino G, Guidetti C, et al. Laparoscopic versus robotic surgery for hepatocellular carcinoma: the first 46 consecutive cases. J Surg Res 2017;217:92-9.

2. Wakabayashi G, Cherqui D, Geller DA, et al. Recommendations for laparoscopic liver resection: a report from the second international consensus conference held in Morioka. Ann Surg 2015;261:619-29.

3. Abu Hilal M, Aldrighetti L, Dagher I, et al. The
Southampton Consensus Guidelines for Laparoscopic Liver Surgery: From Indication to Implementation. Ann Surg 2018;268:11-8.

4. Magistri P, Carrano FM, Guidetti C, et al. Challenges of a minimally invasive approach to posterior liver segments. Laparosc Surg 2018;2:26.

5. Ban $\mathrm{D}$, Tanabe $\mathrm{M}$, Ito $\mathrm{H}$, et al. A novel difficulty scoring system for laparoscopic liver resection. J Hepatobiliary Pancreat Sci 2014;21:745-53.

6. Halls MC, Berardi G, Cipriani F, et al. Development and validation of a difficulty score to predict intraoperative complications during laparoscopic liver resection. Br J Surg 2018;105:1182-91.

7. Lee KF, Cheung YS, Chong CC, et al. Laparoscopic and robotic hepatectomy: experience from a single centre. ANZ J Surg 2016;86:122-6.

8. Di Benedetto F, Magistri P, Ballarin R, et al. Ultrasound-Guided Robotic Enucleation of Pancreatic Neuroendocrine Tumors. Surg Innov 2019;26:37-45.

9. Woo Y, Choi GH, Min BS, et al. Novel application of simultaneous multi-image display during complex robotic abdominal procedures. BMC Surg 2014;14:13.

10. Cipriani F, Shelat VG, Rawashdeh M, et al. Laparoscopic Parenchymal-Sparing Resections for Nonperipheral Liver Lesions, the Diamond Technique: Technical Aspects, Clinical Outcomes, and Oncologic Efficiency. J Am Coll Surg 2015;221:265-72.

Cite this article as: Di Benedetto F, Tarantino G, Magistri P. Chasing the right path: tips, tricks and challenges of robotic approach to posterior segments. HepatoBiliary Surg Nutr 2019;8(5):512-514. doi: 10.21037/hbsn.2019.04.07 